\title{
Le traitement par substitution devant l'agénésie des incisives latérales supérieures
}

\section{The treatment by substitution in front of the agenesie of the superior side incisors}

\section{Résumé}

\section{MOTS-CLEFS :}

- Agénésie des incisives latérales, traitement par substitution,

fermeture des espaces

\section{KEYWORDS:}

- Agenesie of the side incisors, treatment (processing) by substitution, closure (lock) of spaces
L'absence des incicives latérales maxillaires crée un problème esthétique majeur et pose très souvent des difficultés quant à la décision thérapeutique.

L'orthodontiste doit choisir entre deux solutions : l'ouverture pré-prothétique d'espaces ou la fermeture d'espaces remplaçant les latérales manquantes par les canines.

$\grave{A}$ travers un cas clinique, cet article abordera la solution de fermeture des espaces qui reste une solution plus agréable qu'une réalisation prothétique lorsqu'elle répond à des critères particuliers de finition.
Abstract

Missing maxillary lateral incisors create a major esthetic problem. Therapeutic decision is frequently difficult to take. Two treatment approaches are possible: creating adequate space to prosthetically replace the missing lateral incisors or closing the spaces and replacing the missing lateral incisors by the conines.

Through a case report, this article will approach the solution of space closure which is more pleasant than a prosthetic rehabilitation when she answers to the orthodontic finishing criteria.

$\operatorname{AOS} n^{\circ} 273-2015$

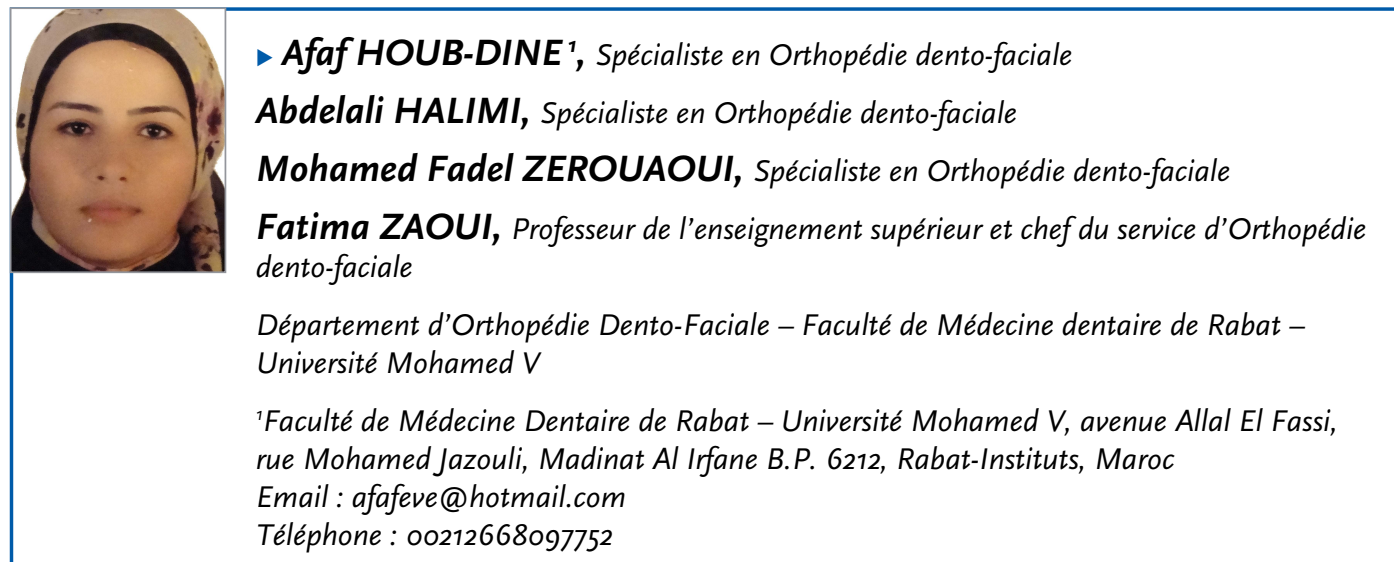

\section{INTRODUCTION}

L'agénésie de l'incisive latérale supérieure représente une bonne part des agénésies dentaires et pose très souvent un problème quant à la décision thérapeutique qui reste un compromis raisonnable entre l'exigence esthétique, les contraintes fonctionnelles, le schéma squelettique et dentaire et l'attente de notre patient.
Si l'on excepte l'abstention thérapeutique lorsque les espaces sont régulièrement répartis et qu'il n'y a pas de doléance esthétique, les deux possibilités thérapeutiques communément utilisées sont soit la réouverture des espaces pour recevoir une prothèse dento-portée ou implanto-portée, soit plutôt la fermeture orthodontique de ces espaces par la mise en place des canines en situation de substitution des incisives latérales manquantes. 


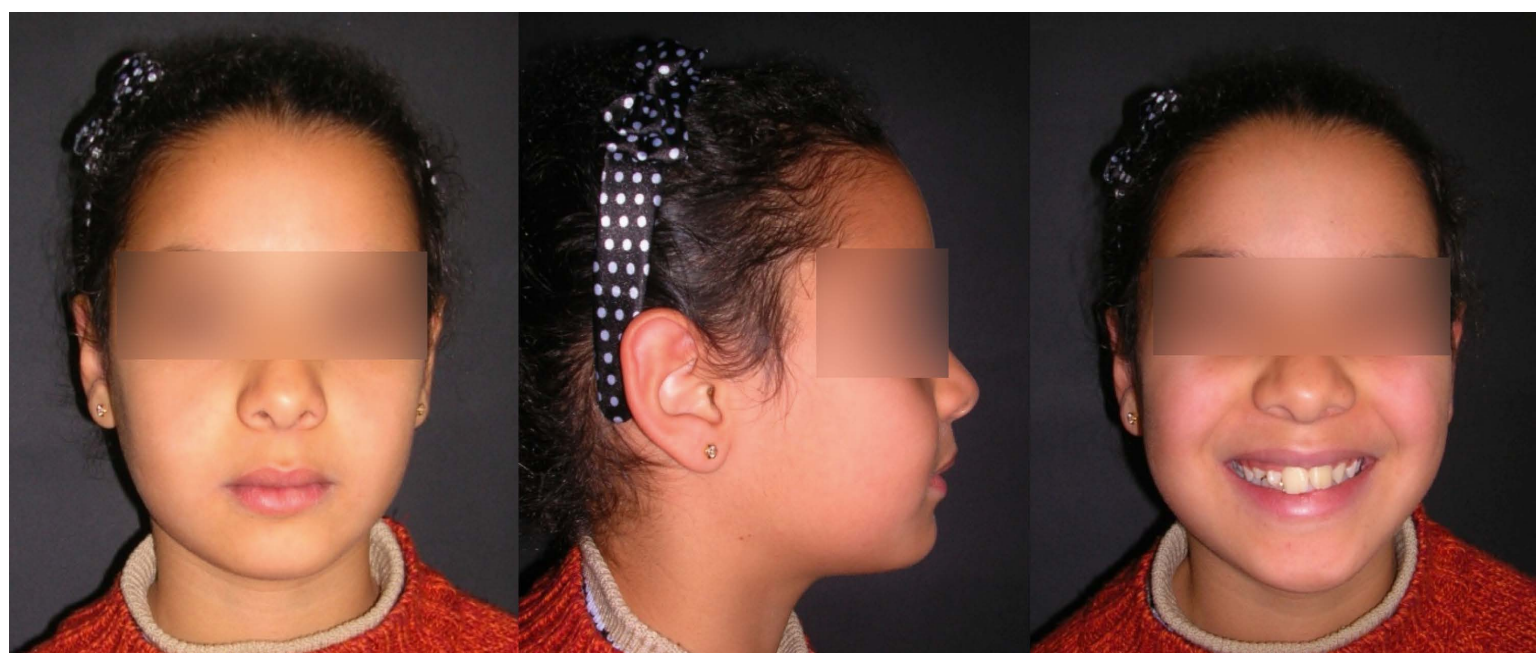

$\triangle$ Fig. 1 : face, profil et sourire avant traitement.

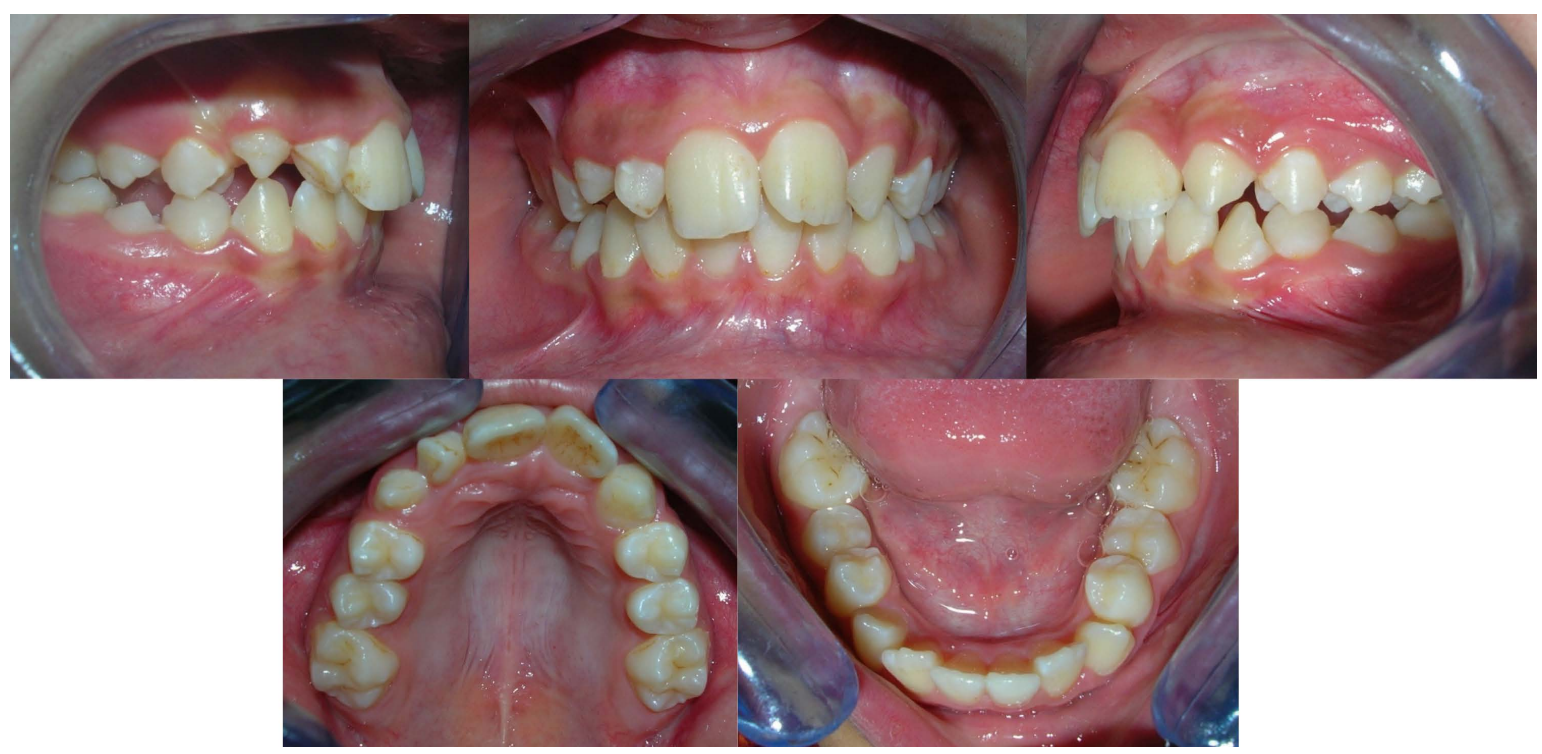

$\triangle$ Fig. 2 : vues endobuccales avant traitement.

L'avantage de cette dernière option thérapeutique est le coût faible et la courte durée de traitement puisque l'acte prothétique est supprimé, mais cette solution requiert une parfaite maîtrise de la technique orthodontique multi-attache.

Le but de cet article est d'illustrer ce concept thérapeutique de première intention par un cas clinique «type » traité au service d'orthopédie dento-faciale CCTDRabat, et de discuter les résultats thérapeutiques de ce traitement avantageux.

\section{CAS CLINIQUE TYPE}

Une jeune patiente âgée de 11 ans s'est présentée à la consultation avec ses parents pour motif esthétique. L'analyse des documents cliniques (Fig. 1, 2) et radiographiques (Fig. 3) de cette patiente a permis d'établir le diagnostic suivant :
I Une classe I squelettique hyperdivergente, I Une DDM de $19 \mathrm{~mm}$,

I Une classe II occlusale de $4 \mathrm{~mm}$

I Déviation de la médiane supérieure de $2 \mathrm{~mm}$

1 Une supraclusion incisive

I Une agénésie de la 22 et de la 12 avec une dent surnuméraire (odontome) (Fig. 2,3).

Une analyse minutieuse des possibilités thérapeutiques a conduit au protocole suivant :

I Un traitement orthodontique multi-attaches avec extraction de 34 et 44 pour corriger la DDM mandibulaire,

I Extraction de la dent surnuméraire (odontome) et de la 12 pour symétriser le problème et permettre la correction de la dysmorphose antérieure, les espaces antérieurs étant fermés

I Une coronoplastie des canines

Ce modelage partiel des canines a été fait en plusieurs étapes au fur et à mesure que se sont établis les 

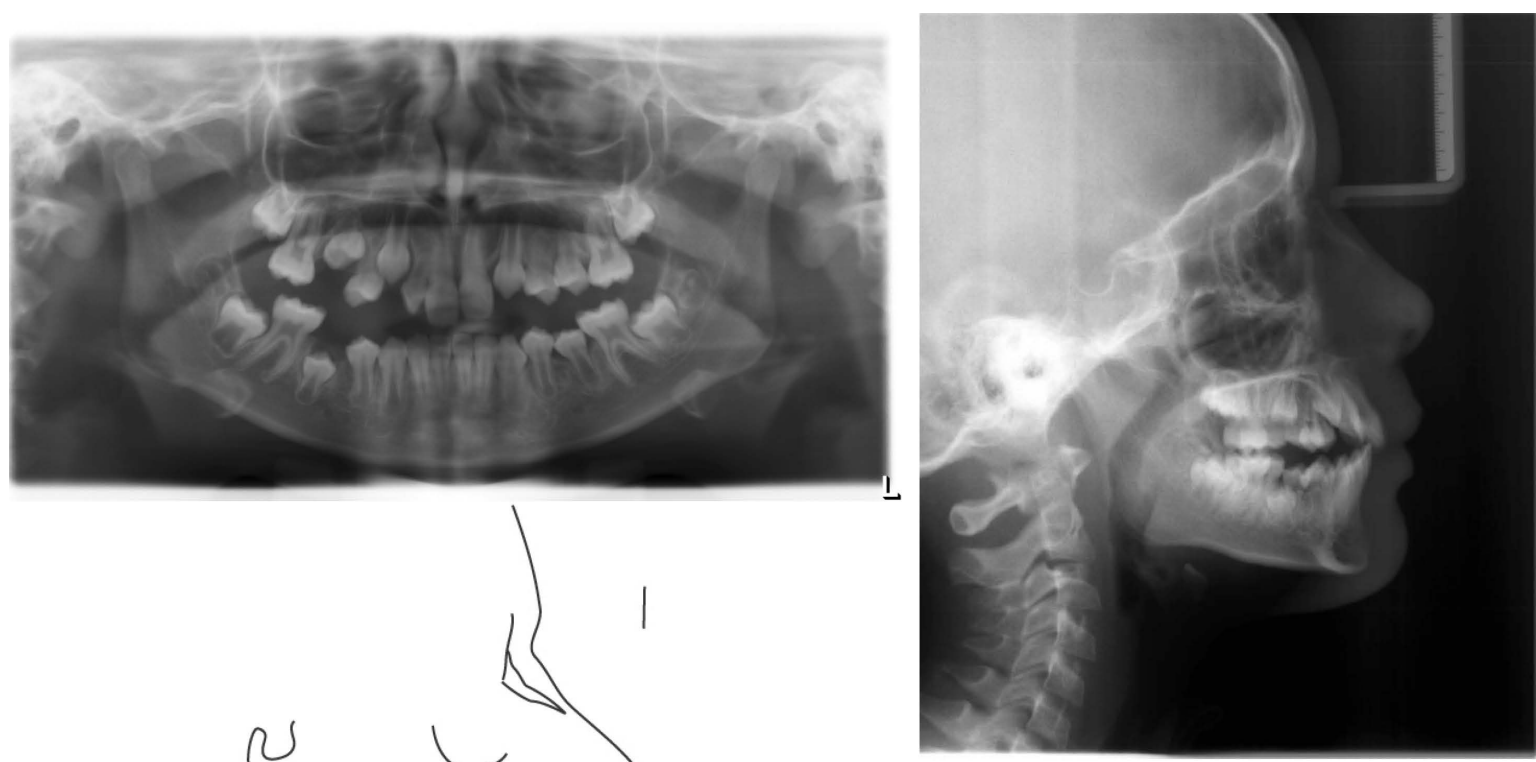

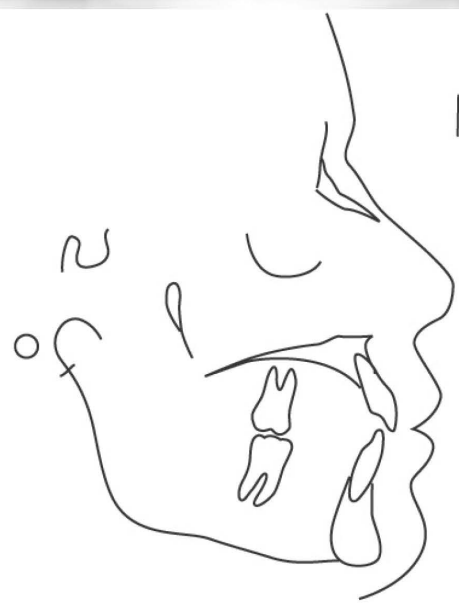

contacts des faces des canines avec les collatérales et les antagonistes.

La diminution des faces proximales des canines a été réalisée en premier permettant d'une part la diminution du diamètre mésio-distal pour se rapprocher des dimensions d'une incisive latérale, d'autre part le rapprochement du point de contact mésial au bord occlusal.

Une diminution légère de la face palatine a permis d'éviter les traumas occlusaux sur les incisives inférieures.

L'aspect esthétique de la ligne du sourire a été obtenu grâce à l'élimination de la pointe cuspidienne et la création d'un bord incisif.

Après débaguage (Fig. 8, 9), nous avons procédé à la dernière intervention qui consiste en l'aplatissement de la face vestibulaire par un léger effacement de la convexité.

La mise en place d'une contention collée rétro-incisive supérieure est le garant d'une stabilité a court et à long termes afin d'éviter le risque de réouverture des espaces de 12 et 22. (Fig. 8)

\section{DISCUSSION}

La mise en place des canines en incisives latérales avec fermeture des espaces antérieurs dans ce cas clinique a permis de concilier au mieux nos objectifs esthétiques fixés et la satisfaction des parents de notre jeune patiente (Fig. 4, 5).

Plusieurs éléments nous ont incité à opter pour un traitement par substitution, en plus du profil équilibré de notre jeune patiente, la fermeture des espaces va dans le sens de la correction de la Classe II. D'autre part, la morphologie de la 12 n'est pas favorable et nécessiterait de toute façon une reconstruction prothétique. De plus, la correction de la DDM nécessitait des extractions mandibulaires.

En dehors des contre-indications liées à la présence d'une supraclusion, d'un déséquilibre occluso-fontionnel et d'une classe III, la fermeture des espaces est indiquée dans les cas de biproalvéolie, DDM et/ ou une classe II, ce qui nécessite souvent l'avulsion de deux prémolaires mandibulaires pour corriger la dysharmonie et obtenir une classe I molaire $[2,6,10]$ L'ouverture d'espaces pour les latérales manquantes devrait être considérée si la vestibulisation des incisives maxillaires est indiquée pour réduire une occlusion inversée antérieure ou pour obtenir un meilleur support de la lèvre supérieure. De même en l'absence de DDM avec une occlusion de classe I.

La phase orthodontique du traitement par ouverture de l'espace consiste à maintenir ou à rétablir l'occlusion postérieure, normaliser le recouvrement et le surplomb incisifs, fermer le diastème entre les centrales et distaler les canines de sorte à obtenir un espace pré-prothétique adéquat au niveau des latérales manquantes. 


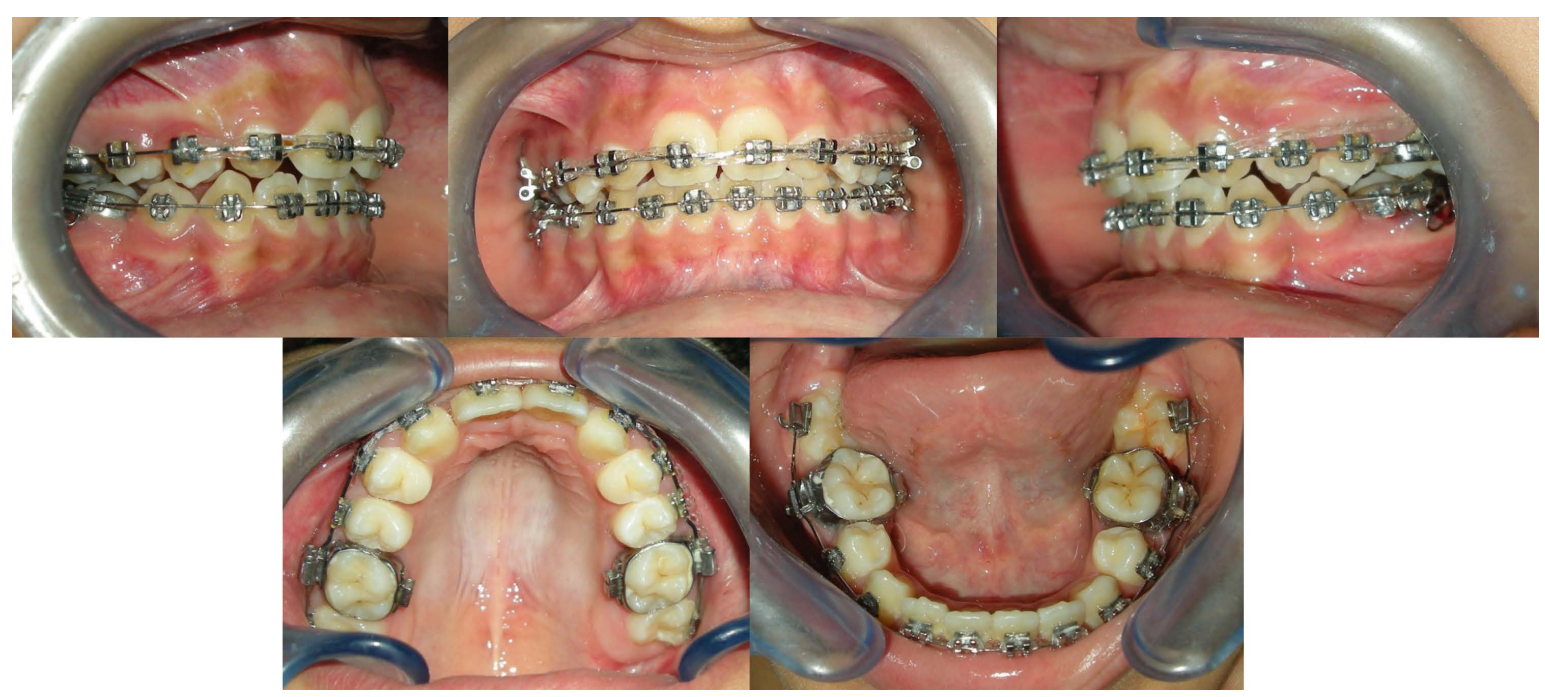

$\triangle$ Fig. 4 : vues endobuccales au cours du traitement orthodontique.
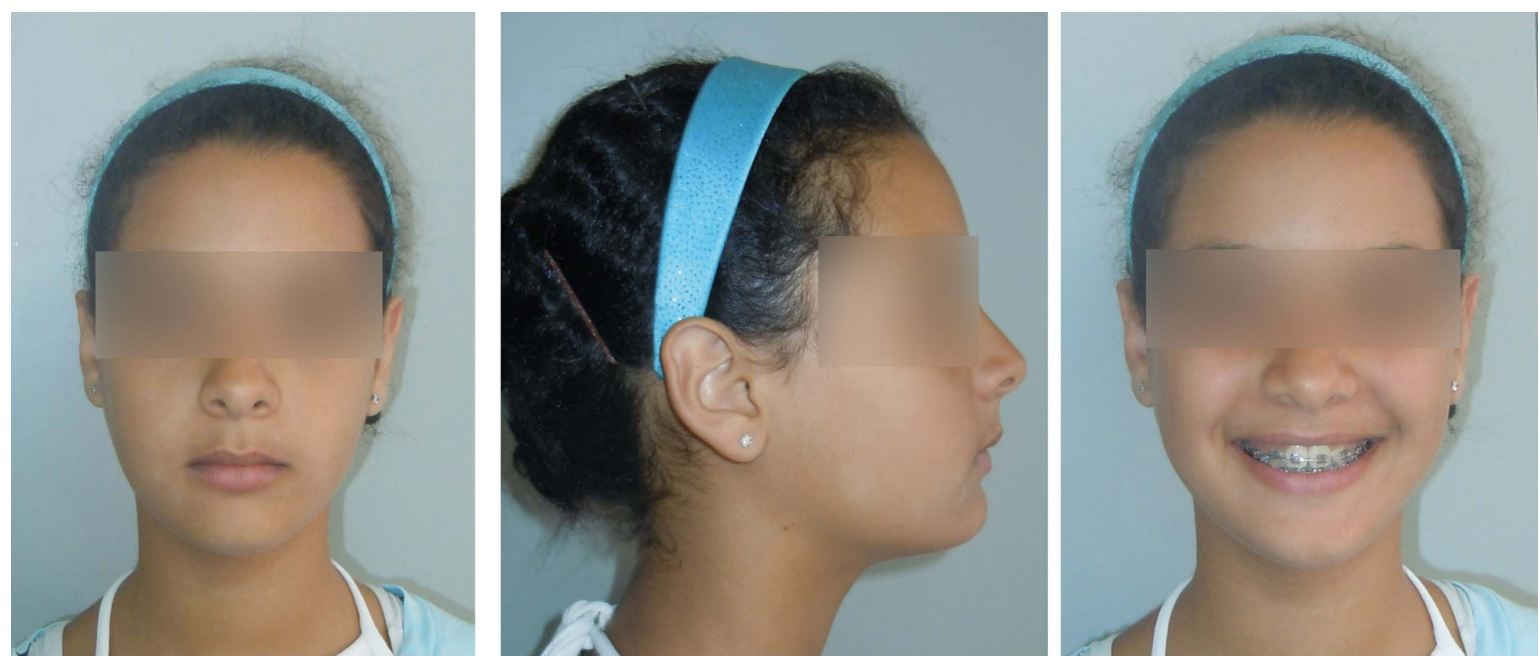

$\triangle$ Fig. 5 : face, profil et sourire au cours du traitement.

Une fois le traitement orthodontique terminé commence une phase de prothèse transitoire de plusieurs années, jusqu’à la fin de la croissance, délicate à gérer. Elle nécessite la collaboration de plusieurs spécialistes, et demande une prothèse de grande qualité. Les implants sont souvent contre-indiqués en raison du volume osseux faible aggravé par l'ouverture de l'espace, et le bridge collé est la solution le plus souvent indiquée [8] Le refus de prothèse et l'absence de motivation pour un traitement pluri-disciplinaire sont des facteurs en faveur d'un traitement purement orthodontique et dont l'avantage principal est la fermeture des espaces. Le traitement se termine réellement à la fin de la période orthodontique. C'est un traitement plus court qui ne nécessite l'intervention que d'un seul spécialiste, en l'occurrence l'orthodontiste $[6,10]$

Par ailleurs, un autre avantage d'une telle option tient surtout au fait que le déficit dentaire est compensé définitivement dès l'adolescence et qu'elle semble bien toléré aussi bien chez l'adulte, l'adulte jeune que l'adolescent contrairement à la solution d'ouverture des espaces avec prothèse implantaire puisque certains adultes jeunes peuvent présenter des remaniements alvéolaires importants et donc l'affirmation de certains auteurs $[5,9,12]$ qui suggèrent de placer les implants à 16 ans chez les filles et à 18 ans chez les garçons apparaît discutable, voire inappropriée.

Des études menées par Thilander et al. [11] et Brugnolo et al. [4] ont souligné la nécessité d'attendre la fin de la croissance chez l'adolescent afin d'éviter qu'une prothèse implanto-portée unitaire ne se retrouve en infraclusion par rapport aux dents naturelles adjacentes avec certainement un décalage vertical associé, préjudiciable sur le plan esthétique.

Cependant, ce choix thérapeutique devra s'accompagner bien souvent de la coronoplastie des canines si leur anatomie est défavorable au plan esthétique : il s'agira de la transformation de la canine en pseudo-latérale 

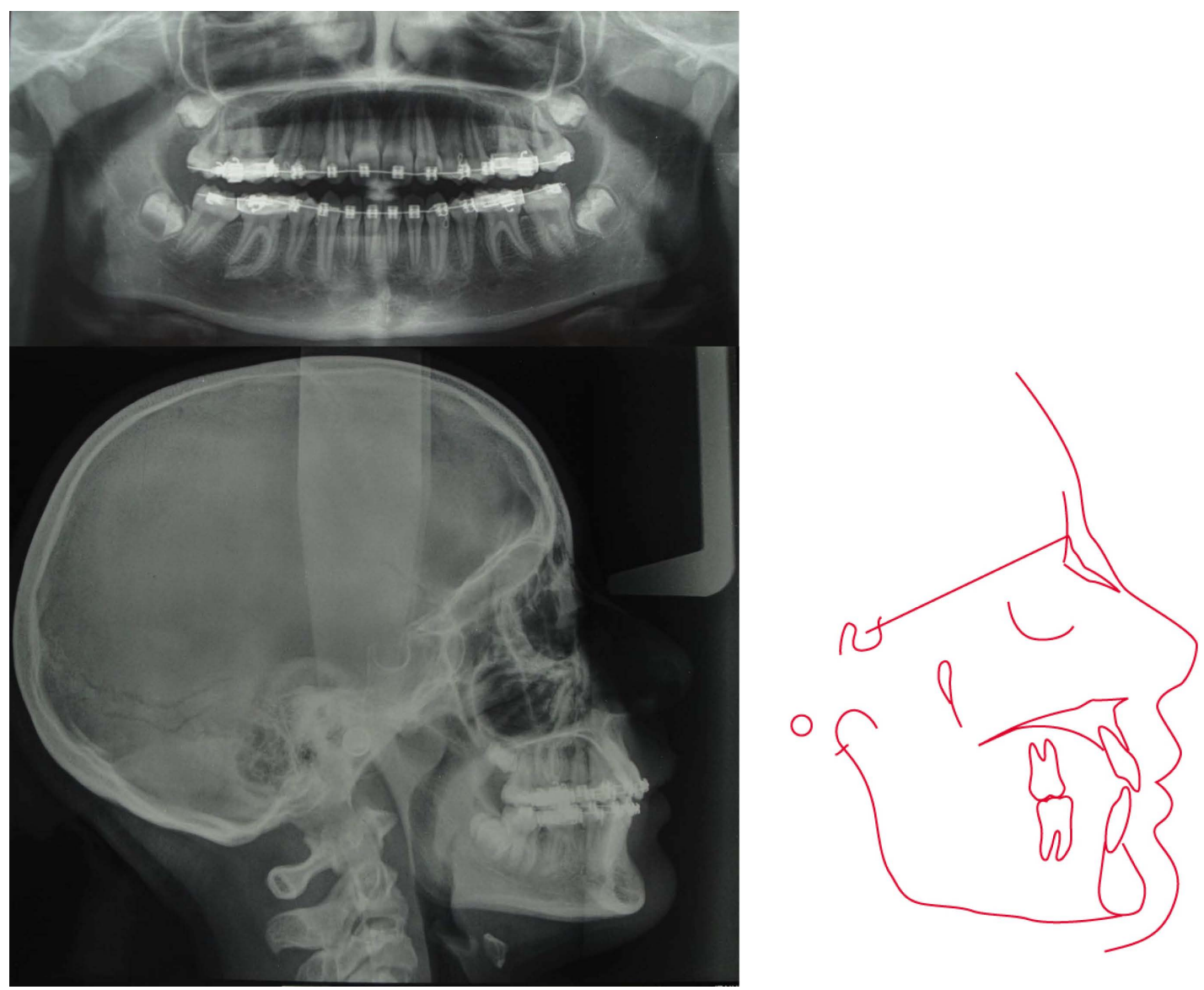

$\triangle$ Fig. 6 : panoramique, téléradiographie et tracé céphalométrique de fin de traitement.
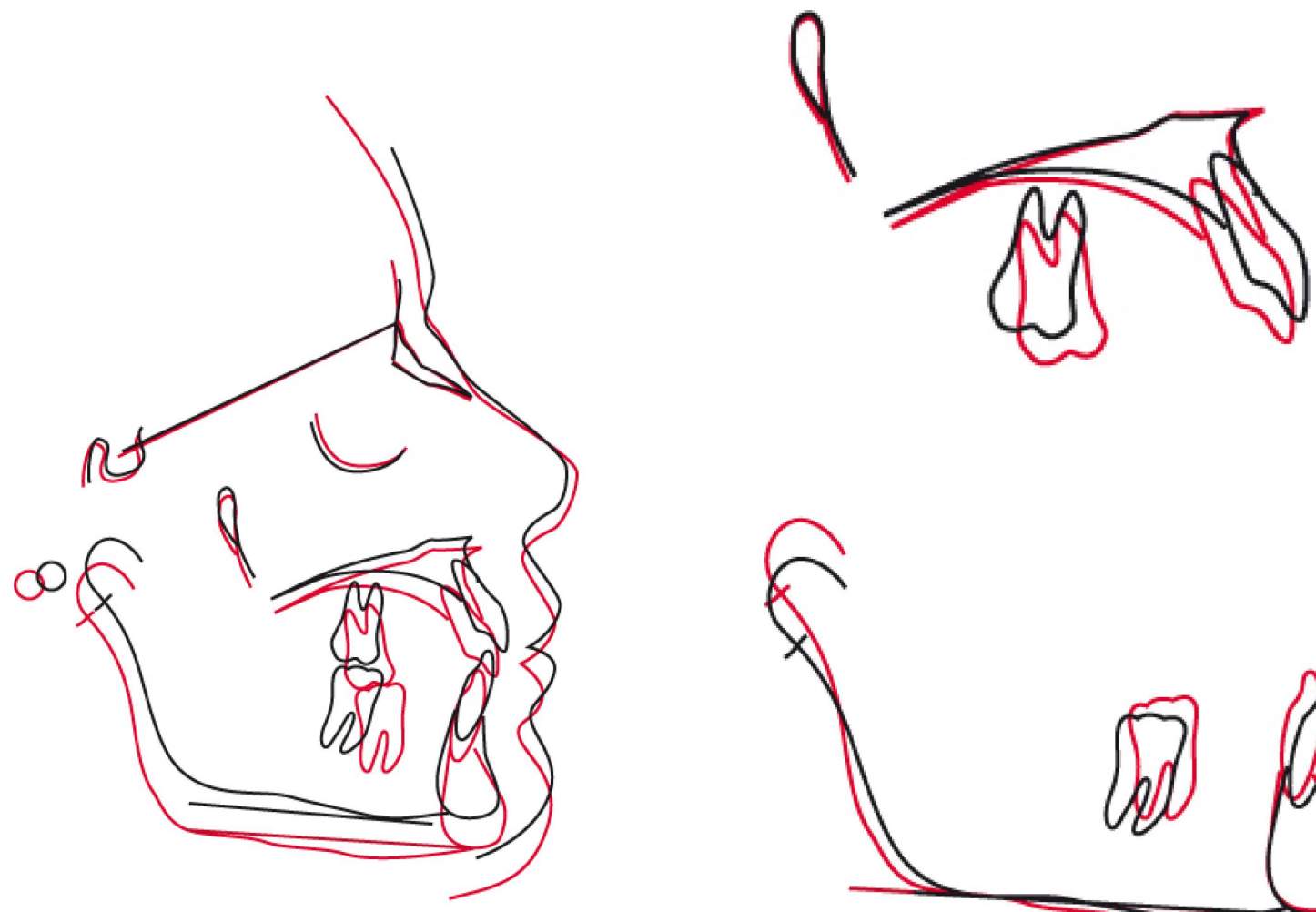

$\triangle$ Fig. 7 : superpositions générales et locales. 

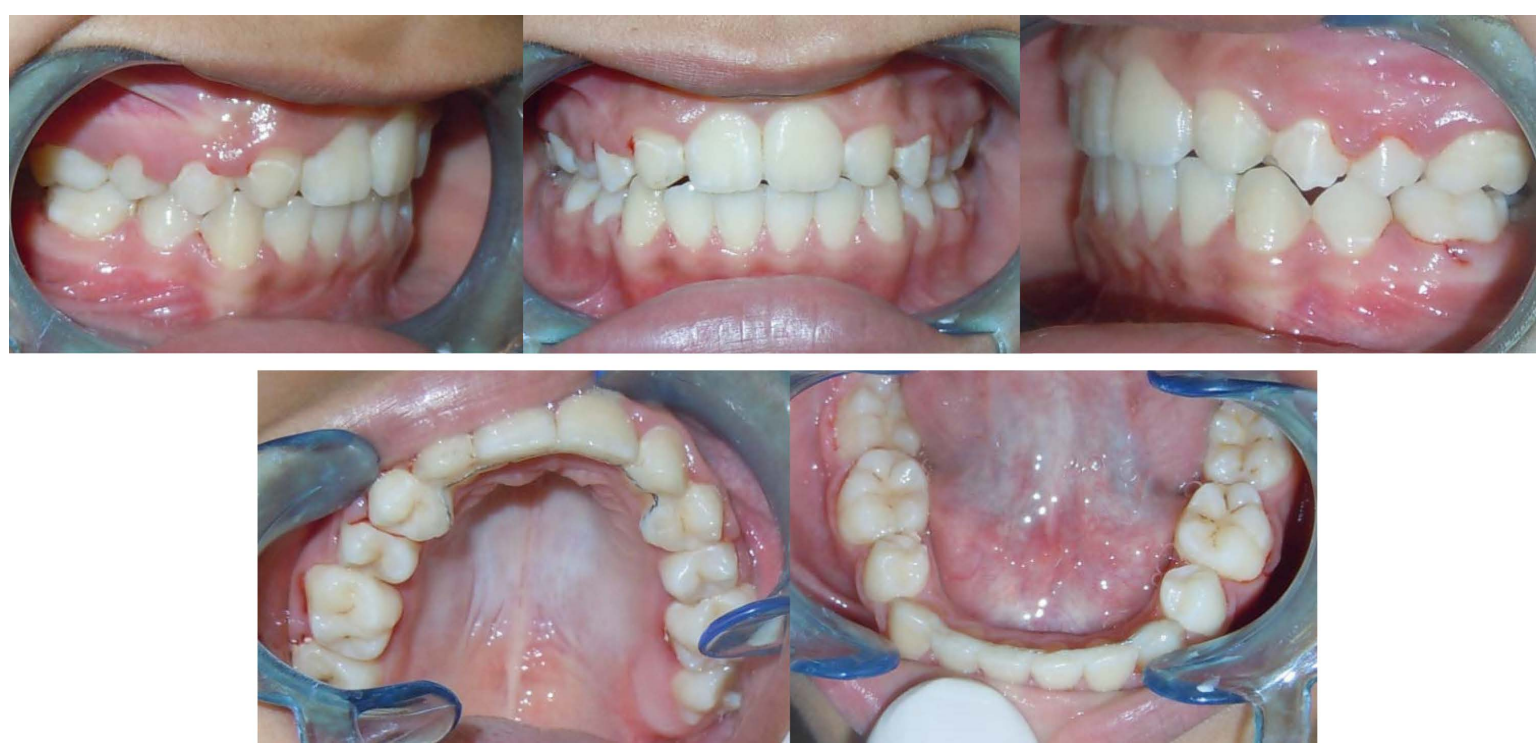

$\triangle$ Fig. 8 : vues endobuccales de fin de traitement.

par coronoplastie soustractive, mais aussi quelques finitions occlusales :

I Augmentation du torque corono-lingual de la première prémolaire pour éviter que la cuspide linguale ne crée une interférence du côté non travaillant.

\ Légère égression des canines de sorte que leur niveau gingival soit légèrement plus occlusal que celui des centrales

Certaines interventions de chirurgie muco-gingivale peuvent aussi participer à l'amélioration du résultat, harmonisant la ligne des collets [1].

La stabilité de la fermeture d'espace, passé un délai de contention suffisant, n'est guère surprenante puisque la substitution effectuée tend plutôt à surdimensionner le bloc incisivo-canin supérieur [3].

Enfin, rappelons que Robertsson et al. [7] ont mené une étude rétrospective sur 50 patients (36 femmes 14 hommes) présentant 39 agénésies bilatérales. Il est étonnant de constater que $93 \%$ des patients présentant des canines en situation de latérales (fermeture d'espace) sont satisfaits de leur esthétique alors que seulement $65 \%$ des patients reconstruits prothétiquement témoignent de leur satisfaction.

\section{CONCLUSION}

Le coût, la complexité et la durée des traitements des agénésies des incisives latérales par ouverture des espaces et remplacement prothétique ne sont pas toujours adaptés ni acceptés par les patients.

Le traitement par fermeture des espaces est un traitement de substitution qui reste largement tributaire de la phase de finition orthodontique.

La décision d'ouvrir ou de fermer l'espace nécessite une étude minutieuse de l'anatomie des dents, de la ligne $\mathrm{du}$ sourire, de l'occlusion initiale et potentielle, de la situation clinique rencontrée. La gestion thérapeutique de l'agénésie des incisives latérales est un compromis raisonnable entre la fonction et l'esthétique.

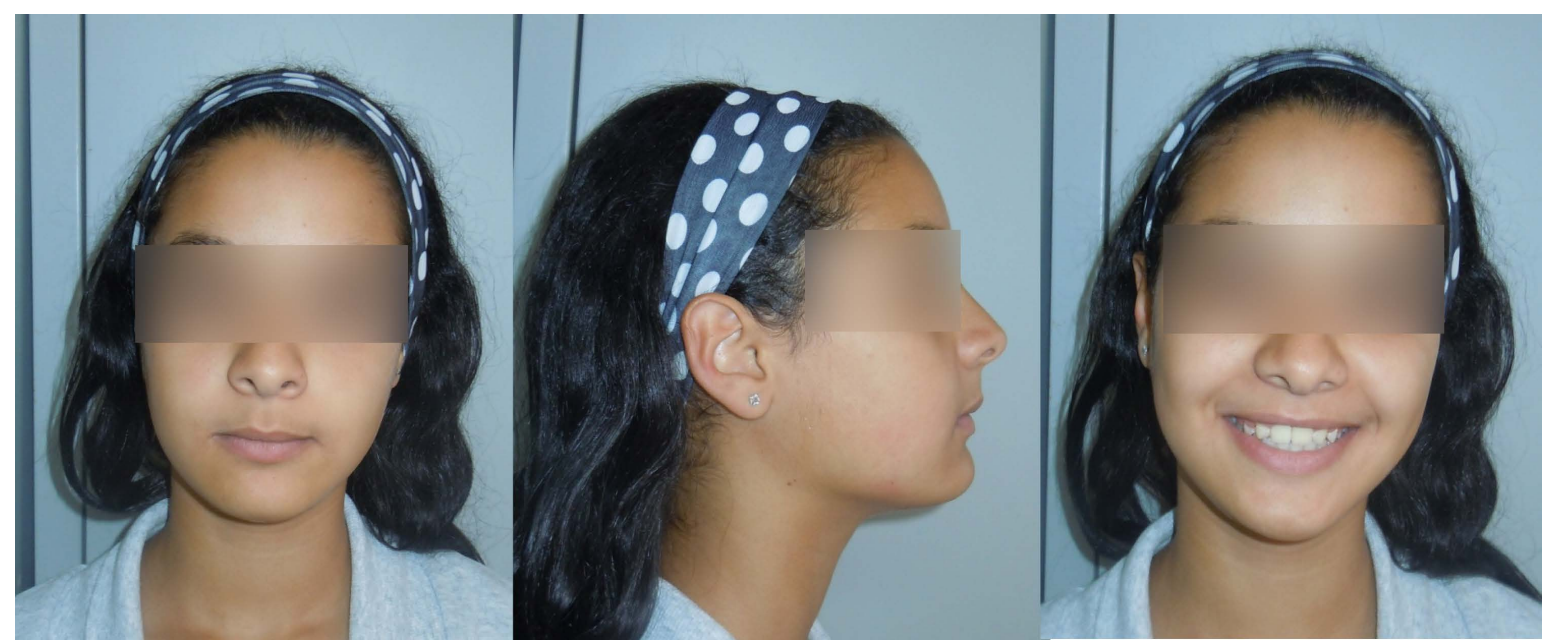

$\triangle$ Fig. 9 : face, profil et sourire de fin de traitement. 


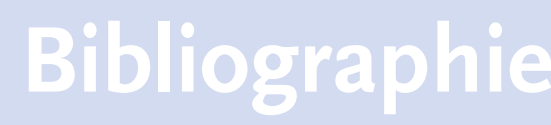

[1] Attia Y. Agénésies d'incisives latérales maxillaires « finition des cas ». Orthod Fr 1984, 55 (2) : 455-465.

[2] Barthelemi S., Jacquelin L-F, Berthet A. Place de la canine dans le traitement des agénésies de l'incisive latérale maxillaire. Rev. Francoph Odontol Pediatr $2009 ; 4$ (1) : 30-40.

[3] Faure J., Baron P., Justumus P. Agénésies des incisives latérales supérieures : évaluation statistique des traitements par substitution. Rev Orthop Dento Faciale 1994 ; 28 : 211-224.

[4] Brugnolo E, Mazzocco C, Gordiol G et al. Clinical and radiographic findings following placement of single tooth implants in young patients - case reports. Int J Periodont Rest Dent 1996;16:421-433.

[5] Kokich VG, Spear FM. Guidelines for managing the orthodontic-restorative patient. Semin Orthod 1997;3(1):3-20.

[6] Martin M. Possibilités thérapeutiques dans le cas d'agénésies d'incisives latérales supérieures. Rev Orthop Dento Faciale 1992 ; $26: 87-97$.

[7] Robertsson S, Mohlin B. The congenitally missing upper lateral incisor; A retrospective study of orthodontic space closure versus restorative treatment. Eur J Orthod 2000 ; 22(6) : 697-710.

[8] Samama Y., Menceur S., Bouniol H. L'agénésie des incisives latérales maxillaires : données actuelles sur les solutions thérapeutiques en cas d'ouverture des espaces. International Orthodontics $2005 ; 3$ : 115-127.

[9] Spear FM, Mathews DM, Kokich VG. Interdisciplinary management of single-tooth implants. Semin Orthod 1997 ; 3(1) : 45-72.

[10] Thierrry M., Granat J., Vermelin L. Les agénésies dentaires : origine, évolution et orientations thérapeutiques. International Orthodontics 2007; 5 : 163-182.

[11] Thilander B, Odman J, Grondahl X, Friberg B. Implants ostéo-intégrés chez l'adolescent. Une solution de remplacement des dents manquantes. Rev Orthop Dento Fac 1996; $30: 381-393$.

[12] Westwood RM, Duncan JM. Implant in adolescents: a literature review and case reports. Int J Oral Maxillofac Implants 1996 ; $11(6): 750-755$. 